\title{
Impact of health education program on diabetic control among diabetic patient managed at diabetic and endocrine center in Bisha, Saudi Arabia.
}

\author{
Abdullah M. AL-Shahrani* \\ Department of Family Medicine, College of Medicine, University of Bisha, Bisha, Saudi Arabia
}

\begin{abstract}
Objective: To assess the impact of diabetic health education on glycemic control for diabetic patients in Bisha province, Saudi Arabia.

Subjects and methods: A prospective study was conducted among adult $\mathbf{4 6 5}$ diabetic patients attending at the Diabetic and Endocrine Center in Bisha, Saudi Arabia, between August 2016 and July 2017. Personal and demographic characteristics were recorded and then each patient underwent 30 min regular diabetic education program every two months along one-year period. Two blood samples were taken (immediately and after one year) from each patient during program to estimate the HbA1c level. A paired $\mathrm{t}$-test was used to compare the level of $\mathrm{HbA1c}$ before and after health education program.

Results: $\mathbf{4 6 5}$ patients were enrolled in our health education program. The majority of them was Saudi $(93 \%)$, married $(86.9 \%)$ and educated with undergraduate level $(94.5 \%)$. There was significant improvement in glycemic control after one year completion of 30 min diabetic education program (HbA1c 10.41 \pm 1.89 to $8.22 \pm 1.68(P<0.012)$ ).

Conclusion: This study demonstrates the effectiveness of regular diabetes health education on glycemic control. We recommend implementation of such education program on regular base as a part for type 2 diabetes control strategic program.
\end{abstract}

Keywords: Health education, Type 2 diabetes, Glycemic control, Bisha, Saudi Arabia.

Accepted on April 28, 2018

\section{Introduction}

Diabetes Mellitus (DM) is a major global health problem, which has been shown dramatic increase during the last three decades. In 2000, the prevalence of DM was $2.8 \%$, which estimated to be increased to $4.4 \%$ in 2030 that mean increase from 171 million to 366 million [1].

Recent report from World Health Organization (WHO) estimated that 7 million of Saudis are struggling with burden of DM. Despite the importance and strong evidence of early diagnosis and treatment of DM, 28\% were unaware of having DM [2].

In Saudi Arabia, the prevalence based on World Population as standard was $18.3 \%$ for DM and $11.9 \%$ for prediabetes. The prevalence of DM and prediabetes increased with age. Of people, aged $\geq 50$ y $46 \%$ of men and $44 \%$ of women had DM. DM was also associated with and family history of dyslipidemia in women, cardiovascular disease in men, and with hypertension, dyslipidemia and family history of diabetes in both sexes [3].

To my knowledge, there is a gap in studies regarding the effects and impact of diabetes health education on glycemic control in Saudi Arabia as well as in many Arabic countries. It is a national policy to study such chronic disease in Saudi population, a growing population with sedentary life style facing many challenges in this regards. This study was designed and conducted to assess the impact of diabetic health education on glycemic control for diabetic patients in Bisha province, Saudi Arabia.

\section{Patients and Methods}

This was a prospective study, conducted among 465 diabetic patients at the Diabetic and Endocrine Center in Bisha province, Saudi Arabia, between August 2016 and July 2017.

The sample size for the study was determined by assuming the prevalence of poor glycemic control in Saudi Arabia was $67.7 \%$ as previously described [4]. We used Epi Info (version 7.2) to calculate the sample size with the confidence interval of $95 \%$ and a $4 \%$ of acceptance margin of error. Based on these sampling, 465 individuals were selected to represent 4100 patients enrolled in Diabetic and Endocrine Center in Bisha.

The inclusion criteria for participants were; adult patients, type 2 diabetes, regularly attended, completed more the four visits at the end of program and referred from primary health care where they not responded to diet and exercise and started medication. The exclusion criteria included; patients participated in previous diabetes education program, having 
gestational diabetics or medically unstable and those with complication.

\section{Collection of data}

Data was collected using a common data form for the following items: personal and demographic characteristic of each patients were collected including, age, gender, marital status, level of education, type of therapy. Two blood samples were taken from each patient during our program to estimate the glycated haemoglobin (HbA1c) level. The first sample was collected immediately after the first visit of the participant. While the second sample was taken after his successfully completion of one year of our health educational program. The reference values of these measures were those recommended by the American Diabetes Association, which defined poor glycemic control as $(\mathrm{HbA} 1 \mathrm{c} \geq 7 \%)[5]$.

\section{Intervention}

Each of the participants received a regular individual diabetic education program during every two month scheduled visits. The program of interactive health education was done by multidisciplinary team included; dialectologist, diabetic health educators and dieticians. The approach used was an interactive approach rather than passive listening.

Supporting material was made available and provided to patients, these included self-home blood glucose monitoring logbook and leaflet regarding diabetic control tools.

The sessions was started by brain storming session to explore prior knowledge and practices of participants, in addition to providing room for sharing experience, beliefs, and opinions. The training concerned on the main issues regarding diabetes. Mainly; definition of the disease, hyper and hypoglycemia, complications associated with poor control of glucose levels, role of diet regimen and physical exercise in diabetes control, hypoglycemic agents, associated diseases, chronic complications, dental and foot care, diabetes care during especial gatherings and occasions (Ramadan, Hajj, and travelling). In addition, practical aspects of the training covered insulin injection technique and home-self monitoring of blood glucose.

The turn of each individual comes every two months where a revision and reinforcement of the educational program took place as well as laboratory follow-up of the HbAlc.

The American Diabetes Association (ADA) guidelines were used for diagnosis of diabetes (A1C $\geq 6.5 \%$ ), prediabetes (A1C 5.7-6.4\%), or normal glycemia (A1C $\leq 5.6 \%)[6]$.

\section{Statistical analysis}

Data entry and analysis were carried out using Statistical Program for the Social Sciences (SPSS). Patient demographic characteristics were analysed using descriptive statistics in terms of frequency, percentage, mean and standard deviation. A paired t-test was used to compare the level of HbA1c before and after health education. A p-value less than 0.05 was selected for statistical significance. ANOVA test was used to measure the changes in the means of study parameters.

\section{Ethical considerations}

All the necessary official permissions were fully secured before data collection. The collected data were kept strictly confidential and used only for research purpose. All patients agreed to participate in the study had signed an informed consent and ethical approval was obtained from research ethical committee in College of Medicine, University of Bisha (ethical approval number 418062016).

\section{Results}

In this study a total of adult 465 diabetic patients attending at the Diabetic and Endocrine Center in Bisha, Saudi Arabia were enrolled in a well-organized health education program, they continued the program of one year and those who discontinued were withdrawal from the analysis $(12 / 477(2.5 \%))$. The primary outcome was to assess the impact of diabetic health education on glycemic control for diabetic patients. The average age of them was $52 \mathrm{y}$, ranging from 30 to $90 \mathrm{y}$. Of these 465 patients, $267(57.4 \%)$ were females and $198(42.7 \%)$ were males. The majority of patients were Saudi (93\%), married $(86.9 \%)$ and educated with undergraduate level (94.5\%). About $68 \%$ of the patients were on oral hypoglycemic agents, $30 \%$ were on a combination of oral hypoglycemic agents and insulin and only $2 \%$ were on insulin alone (Table 1).

The values of HbA1c were significantly $(p<0.012)$ decreased after the successful completion of the health education program while insignificant changes in medication reported ( $P$ value $0.63)$. Baseline body weight was $75.51 \pm 11.13$ kilograms, with non-significantly different between males and females ( $\mathrm{P}$ value 0.75). However after the education program, there was significant difference $(\mathrm{P}$ value $<0.0002)$ (Table 2$)$.

Table 1. Characteristics of adult patients $(n=465)$ with type 2 diabetes those are participated in health education program at Diabetic and Endocrine Center in Bisha, Saudi Arabia.

\begin{tabular}{ll}
\hline Character & Number of patients (\%) \\
\hline Age & $145(31.2)$ \\
\hline$<45$ y & $320(68.8)$ \\
\hline$>45$ & \\
\hline Gender & $267(57.4)$ \\
\hline Female & $198(42.7)$ \\
\hline Male & \\
\hline Nationality & $432(93)$ \\
\hline Saudi & $33(7)$ \\
\hline Non-Saudi & \\
\hline Marital status & \\
\hline
\end{tabular}




\section{center in Bisha, Saudi Arabia}

\begin{tabular}{ll}
\hline Married & $404(86.9)$ \\
\hline Non-married & $61(13.1)$ \\
\hline Education status & $17(3.5)$ \\
\hline Illiterate & $439(94.5)$ \\
\hline Undergraduates & $9(2)$ \\
\hline Graduates & \\
\hline Type of therapy & $316(68)$ \\
\hline Oral hypoglycemic agents only & $140(30)$ \\
\hline Oral hypoglycemic agents plus Insulin & $9(2)$ \\
\hline Insulin only & \\
\hline
\end{tabular}

Table 2. Comparison of the mean HbAlc level of diabetic patients before and after completion of one year of health education.

\begin{tabular}{llll}
\hline Test & Before & After one year & P value \\
\hline HbA1c & $10.41 \pm 1.89$ & $8.22 \pm 1.68$ & $<0.012$ \\
\hline Body weight & $78.72(13.77)$ & $75.51 \pm 11.13$ & $<0.0002$ \\
\hline
\end{tabular}

\section{Discussion}

The issue of patient's education is so important for us in Saudi Arabia, many programs addressed this sensitive and important intervention, the importance of patient education has been known and emphasized as early as the late eighteenth century. In the early 1920's Joslin stated that "the diabetic who knows the most, lives the longest" [7]. This was confirmed by recent data that patient education has great efficiency in improving metabolic control [8].

This study was perfectly assessed the impact of diabetic health education on glycemic control for diabetic patients in Bisha province, Saudi Arabia. The study results demonstrated that a regular $30 \mathrm{~min}$ education program is an effective approach in improving glycemic parameters among type 2 diabetes patients after one year of the education program. This achievement of the program possibly due to the regularity of the session time and duration and contents. Adherent to the designed program was of great benefit and lead to strong results. Similar findings have been reported that patient education on lifestyle modifications result in a better control of blood glucose [9].

It was found that on average the glycemic reduction was around $20 \%$ in glycemic control in the current study. Close to this result was that reported by Selea et al. where up to $15 \%$ reduction was achieved after six months of printed material diabetic education [10].

From these finding it was obvious that regular educational sessions that will organize and has a good follow-up are needed to achieve better glycemic control. The protocol used for management was effective and good diabetic education has long been acknowledged as essential in the maintenance of good glycemic control. It is widely accepted as the cornerstone of successful diabetes management [11]. Since diabetes mellitus is associated with high rates of acute and chronic medical, social and psychological problems, the reduction and prevention of these problems cannot be achieved without health education to the patients and their involvement in caring for themselves [12]. Studies on the impact of diabetes health education on glycemic control in Saudi Arabia are very few, and almost all of these studies were conducted to determine the role of knowledge and attending the education program on patient's diabetes control $[13,14]$.

The current study revealed the education program has effect significantly in HA1C reduction which indicating of good in glycemic control. This findings examining how best to deliver diabetes education and skills training and provide important insight into effective strategies to improve metabolic control. This concept has been describe and supported by American an ongoing 4-year randomized clinical trial study [15] this comparable with another study conducted a high quality, pragmatic trial of structured education alongside standard care in diabetes clinics [16]. And positively supported with multicenter randomized controlled trial findings [17].

After the education program, there was significant difference regarding body weight $(\mathrm{P}$ value $<0.0002)$. As mentioned in a study from China that revealed a reduction in the weight after adopting intensive educational program [18].

\section{Conclusion}

This study demonstrates the effectiveness of regular diabetes health education on glycemic control. On average, the glycemic reduction was around $20 \%$ in glycemic control. Regular educational sessions that will organize and has a good follow-up are needed to achieve better glycemic control. The recommendation of implementation of such education program on regular base should take place as an strategic program.

\section{Acknowledgement}

Special thanks to the administration and staff of the Diabetic and Endocrine center in Bisha, in facilitating this work. This work was financially supported by College of MedicineUniversity of Bisha Kingdom of Saudi Arabia.

\section{Conflict of Interest}

Author declared no conflict of interest

\section{References}

1. Wild S, Roglic G, Green A, Sicree R, King H. Global prevalence of diabetes: estimates for the year 2000 and projections for 2030. Diabetes Care 2004; 27: 1047-1053

2. Al-Nozha MM, Al-Maatouq MA, Al-Mazrou YY, AlHarthi SS, Arafah MR, Khalil MZ, Khan NB, Al-Khadra A, Al-Marzouki K, Nouh MS, Abdullah M, Attas O, AlShahid MS, Al-Mobeireek A. Diabetes mellitus in Saudi Arabia. Saudi Med J 2004; 25: 1603-1610.

3. Suhad M. Bahijri, Hanan A. Jambi, Rajaa M. Al Raddadi, Gordon Ferns, and Jaakko Tuomilehto. The prevalence of 
diabetes and prediabetes in the adult population of Jeddah, Saudi Arabia-a community-based survey. PLoS One 2016; 11: 0152559.

4. Stratton IM, Adler AI, Neil HA, Matthews DR, Manley SE, Cull CA, Hadden D, Turner RC, Holman RR. Association of glycaemia with macrovascular and microvascular complications of type 2 diabetes (UKPDS 35): prospective observational study. BMJ 2000; 321: 405-412.

5. American diabetes association. Standards of Medical Care in Diabetes-2017. Abridged for primary care providers. Clin Diabetes 2016; 35: 5-26.

6. American Diabetes Association. Standards of medical care in diabetes-2015. Abridged for Primary Care Providers. Clinical Diabetes 2015; 33: 97-111.

7. Al-Rasheedi AA. The role of educational level in glycemic control among patients with type II diabetes mellitus. Int J Health Sci (Qassim) 2014; 8: 177-187.

8. American Diabetes Association. Standards of medical care in diabetes 2011. Diabetes Care 2011; 34: 11-61.

9. El-Zubier AG, Aladin A, Al-Amri JB, Al-Haraka EA and Abu-Samara IO. Self-care, self-reliance and knowledge of diabetes among diabetes in Qassim region. Saudi Med J 1996; 17: 598-603.

10. Iftikhar U, Tahir JA, Abdur RAK, Rahila I. Diabetes education: its effects on glycemic control. Ann Saudi Med 2001; 21: 1-2.

11. Norris SL, Lau J, Smith SJ, Schmid CH, Engelgau MM. Self-management education for adults with type 2 diabetes: a meta-analysis of the effect on glycemic control. Diabetes Care 2002; 25: 1159-1171.

12. Uusitupa MI. Early lifestyle intervention in patients with non-insulin-dependent diabetes mellitus and impaired glucose. Ann Med 1996; 28: 445-449.

13. Selea A, Sumarac-Dumanovic M, Pesic M, Suluburic D, Stemenkovic-Pejkovic D, Cvijovic G, Micic D. The effects of education with printed material on glycemic control in patients with diabetes type 2 treated with different therapeutic regimens. Vojnosanit Perl 2011; 68: 676-683.
14. Badedi M, Solan Y, Darraj H, Sobai A, Mahfouz M, Alamodi S, Alsabaani A. Factors associated with longterm control of type 2 diabetes mellitus. J Diab Res 2016; 8.

15. Williams JS, Lynch CP, Knapp RG, Egede LE. Technology-Intensified Diabetes Education Study (TIDES) in African Americans with type 2 diabetes: study protocol for a randomized controlled trial. Trials 2014; 15 : 460.

16. Christie D, Thompson R, Sawtell M, Allen E, Cairns J, Smith F, Jamieson E, Hargreaves K, Ingold A, Brooks L, Wiggins M, Oliver S, Jones R, Elbourne D, Santos A, Wong IC, ONeill S, Strange V, Hindmarsh P, Annan F, Viner R. Structured, intensive education maximising engagement, motivation and long-term change for children and young people with diabetes: a cluster randomised controlled trial with integral process and economic evaluation-the CASCADE study. Health Technol Assess 2014; 18: 1-202.

17. Wild SH, Hanley J, Lewis SC, McKnight JA, McCloughan LB, Padfield PL, Parker RA, Paterson M, Pinnock H, Sheikh A, McKinstry B. Supported telemonitoring and glycemic control in people with type 2 diabetes: the telescot diabetes pragmatic multicenter randomized controlled trial. PLoS Med 2016; 13: 1002098

18. Li Y, Xu M, Fan R, Ma X, Gu J, Cai X, Liu R, Chen Q, Ren J, Mao R, Bao L, Zhang Z, Wang J, Li Y. The effects of intensive nutrition education on late middle-aged adults with type 2 diabetes. Int $\mathrm{J}$ Environ Res Public Health 2016; 13: 897.

\section{*Correspondence to}

Abdullah M. AL-Shahrani

Department of Family Medicine

College of Medicine

University of Bisha

Saudi Arabia 